\title{
Analysis of Certain Binary Collision Approximation Closures of the BBGKY Hierarchy
}

\author{
R.F Snider \\ Department of Chemistry, University of British Columbia, \\ Vancouver, Canada V6T 1Z1 \\ W. J. Mullin \\ Department of Physics and Astronomy, University of Massachusetts, \\ Amherst, MA 01003, USA \\ F. Laloë \\ Laboratoire Kastler Brossel de l'ENS, \\ 24 rue Lhomond, F 75005 Paris, France
}

April 15, 2004

\begin{abstract}
Boercker and Dufty (BD) introduce a binary collision approximation into the BBGKY hierarchy in order to describe the low-density behaviour of a quantum gas. Their approach involves the three-particle reduced density operator being approximated in a particular manner that favours correlation between two of the particles, while ignoring the correlation with the third. The tradition of previous derivations, on the other hand, has been to neglect the three-particle term altogether and assume a generalized form of Boltzmann's Stosszahlansatz. Both formalisms reach the same final result: the Waldmann-Snider equation (WS), a quantum version of the Boltzmann equation. We compare the two derivations in two ways: (a) by finding iterated series solutions of the BBGKY hierarchy and of the Boltzmann equation, which demonstrates what kind of terms are absent in the WS and BD approaches in comparison with exact dynamics; (b) by computing an exact
\end{abstract}


correction to the WS equation, which vanishes in the BD scheme provided the mean time of duration of a collision is much shorter than the mean free time between collisions. This correction is shown to be related to the standard three-body collision integral arising in the theory of the density corrections to the Boltzmann equation. We also comment on the related work of Klimontovich, who introduces an approximation analogous to that of Boercker and Dufty. 


\section{Introduction}

Modern "derivations" of the Boltzmann equation and its generalizations are based either on the BBGKY hierarchy ${ }^{1-4}$ or the Green's function method of Kadanoff and Baym ${ }^{5}$. In all cases some closure approximation is required. Most approaches using the BBGKY hierarchy now refer to the Bogoliubov ${ }^{1}$ functional assumption which, in quantum terms, states that all reduced density operators depend on time only via their functional dependence on the singlet density operator. The Green's function approach depends on making an appropriate assumption about the form of the self-energy. The present work is aimed at clarifying the assumptions that are needed to obtain the Boltzmann equation by closing the BBGKY hierarchy without elaborating about its generalization to higher density. It is found that for realistic (soft) potentials the requirement that only isolated binary collisions occur depends crucially on taking the asymptotic time limit of each binary transition superoperator wherever it occurs. This requirement appears little discussed in most discussions about the nature of the Boltzmann equation.

There seems to be three distinct approaches to closing the BBGKY hierarchy. The simplest is to follow Boltzmann's classic approach ${ }^{6}$ and introduce a molecular chaos assumption for the pair density operator. Born and Green $^{2}$ and Kirkwood ${ }^{3}$ followed this procedure classically, with differing rationales for its validity, while Snider ${ }^{7}$ followed this approach to include the quantum treatment of degenerate internal states. Boercker and Dufty8 (BD) introduce a "binary collision approximation" that involves a factorization of the three-particle density operator in such a manner that, formally, only pairs of particles are correlated and interact in the second BBGKY equation, that is, the equation for the pair density operator. For quantum 
systems and restricted to Boltzmann statistics, their approach also results in the Waldmann-Snider (WS) equation ${ }^{9,7}$. The third method, carried out by Cohen $^{10}$ and M.S. Green ${ }^{11}$, depends on comparing the dynamics of a (classical) reduced distribution function for $s$ particles with that of an isolated set of $s$ particles, essentially expanding the dynamics of one in terms of the other. Green ${ }^{11}$ also discusses resumming the expansion so that at arbitrary times during the evolution of the gas, the singlet distribution functions that weight the probability for a particular collision are those associated with particles that have suffered many past collisions. None of these methods are mathematically rigorous and thus all are subject to criticism. Lanford ${ }^{12}$ has made a concerted effort to rigorously derive the Boltzmann equation from the BBGKY hierarchy, but for rigid sphere systems he could only show that the solution of the Boltzmann equation and the evolution of the singlet as deduced from the Liouville equation was valid for times up to a fraction of the mean free time.

The present work was initially motivated by a preprint by Dufty and $\mathrm{Kim}^{13}$ in which they claimed that they had a kinetic equation that "extends the familiar Waldmann-Snider equation to arbitrary length and time scales". Their method is based on the Boercker-Dufty" "binary collision approximation" of the BBGKY hierarchy ${ }^{1-4}$, restricted to the case of Boltzmann statistics. It was in trying to understand the logic behind the BD approach and questioning the validity of their statement that the time integrated form for the singlet density operator as predicted by the exact BBGKY hierarchy was examined and compared to the corresponding equation deduced from the Boltzmann equation. While such expansions are well known and the basis of the work of Cohen ${ }^{10}$ and Green ${ }^{11}$, the present emphasis is different, aimed at a comparison of form rather than attempting to deduce one from the other. 
The differences also emphasize the nature of the assumptions arising in "deriving" the Boltzmann equation from the BBGKY hierarchy following the molecular chaos and Boercker-Dufty approaches.

The organization of the paper is as follows: Section II provides differential and integral forms for the BBGKY hierarchy. These are useful for comparison with the formulas obtained via the various binary collision approximations. An iteration of the time integrated BBGKY hierarchy to yield a formal series solution for the singlet density operator is central to later discussions.

The original derivations of Snider and of BD are examined in Sec. III and a review given of how $\mathrm{BD}$ are able to eliminate the two-body density operator and arrive at the WS equation. We note that both derivations involve further approximations, specifically that 1) a certain infinite time limit of the transition superoperator is taken, and 2) the singlet density operator evolves freely between (binary) collisions. Attention is drawn to these added approximations to emphasize that they are also crucial to the derivation of the Boltzmann equation.

In Sec. IV, we contrast the first few terms of an infinite series solution of the Boltzmann equation, obtained by iteration, with the corresponding iterative solution of the BBGKY hierarchy. We see from this what kinds of terms the Boltzmann equation has neglected in comparison with the exact evolution of the system.

Next, in Sec. V, we compute an additive correction to the WS collision integral. Since this involves three particle contributions to the rate of change for the singlet density operator, the usual approach to deriving the Boltzmann equation using the Stosszahlansatz states that such contributions are to be ignored. Within the BD approximation, the correction is shown to vanish if the $\mathrm{BD}$ approximation is used in its evaluation, provided the infi- 
nite time limit of the transition superoperators are also taken. This shows, in particular, that the $\mathrm{BD}$ approach is a consistent method of deriving the Boltzmann equation. The correction to the WS equation is examined in more detail and to leading order in density it is shown that it is just the standard three-particle collision operator that is found ${ }^{10,14-16}$ in discussions of the density corrections to the Boltzmann equation. It is also related to the terms found to be "missing" in the iterated solution of the Boltzmann equation when compared to the series solution of the BBGKY hierarchy for the one-body density operator.

Klimontovich ${ }^{17}$ also introduces a binary collision approximation which entails a factorization of the three-particle distribution function (for a classical mechanical system) that is very similar in structure to that of BD. The only significant difference is that Klimontovich separates off uncorrelated contributions to the pair and triple distribution functions before carrying out his binary collision approximation. Thus his factorization involves the correlation functions rather than the full distribution functions. Since the formal structure is the same classically and quantally, the comments on his work in Sec. VI are given formally in a quantum language so that a comparison with the Dufty-Boercker-Kim work can be made.

Finally in Sec.VII we summarize the differences between the nature of the solutions of the Boltzmann equation and the BBGKY hierarchy. An interpretation is proposed for rationalizing the physical content of the Boercker Dufty binary collision approximation as well as to why and under what constraints it leads to the Boltzmann equation.

\section{Formal solutions to the BBGKY Hierarchy}


The BBGKY hierarchy ${ }^{1-4}$ constitute a set of coupled equations derived from the quantum Liouville equation. For a quantum system of $\mathrm{N}$ particles having Boltzmann statistics, the equations relating the one particle operator $f_{1}$, the two particle operator $f_{12}$, etc. are

$$
\begin{gathered}
\left(\frac{\partial}{\partial t}+L_{1}\right) f_{1}(t)=n \operatorname{Tr}_{2} \Theta_{12} f_{12}(t) \\
\left(\frac{\partial}{\partial t}+L_{12}\right) f_{12}(t)=n \operatorname{Tr}_{3}\left[\Theta_{13}+\Theta_{23}\right] f_{123}(t) \\
\left(\frac{\partial}{\partial t}+L_{123}\right) f_{123}(t)=n \operatorname{Tr}_{4} \sum_{j=1}^{3} \Theta_{j, 4} f_{1234}(t) \\
\left(\frac{\partial}{\partial t}+L_{1 \ldots s}\right) f_{1 \ldots s}(t)=n \operatorname{Tr}_{s+1} \sum_{j=1}^{s} \Theta_{j, s+1} f_{1 \ldots s+1}(t) \\
\ldots . . . \\
\left(\frac{\partial}{\partial t}+L_{1 \ldots N}\right) f_{1 \ldots N}(t)=0
\end{gathered}
$$

Here $L_{1 \ldots s} A=i / \hbar[H(1, \ldots, s), A]_{-}$is the Liouville superoperator for $s$ particles and $\Theta_{12}=L_{1}+L_{2}-L_{12}$ is $(-i / \hbar)$ times the potential commutator for particles labelled 1 and 2 . For a single particle $L_{1}$ will often be denoted $K_{1}$ for the kinetic contribution to the particle's evolution. A combination of free particle Liouville superoperators will be denoted as $K_{1 \ldots s}=L_{1}+\cdots+L_{s}$. There are several different normalizations that are in use; here we choose to define the density operator $f_{1 \ldots s}(t), s<N$, consistent with the notation of Dufty and $\mathrm{Kim}^{13}$,

$$
n^{s} f_{1 \ldots s}(t)=\frac{N !}{(N-s) !} \operatorname{Tr}_{s+1 \ldots N}\left\{\rho_{1 \ldots N}(t)\right\}
$$

with normalization $\operatorname{Tr}_{1 \ldots N}\left\{\rho_{1 \ldots N}(t)\right\}=1$ and particle density $n=N / V$ having $N$ particles in volume $V$. The factorials introduced in Eq.(6) simplify 
Eqs.(1-5) by properly accounting for the number of possible particles that can interact with a given set of $s$ particles. It also implies that $f_{1 \ldots s}(t)$ is normalized according to

$$
\operatorname{Tr}_{1 \ldots s} f_{1 \ldots s}(t)=V^{s}\left(1-\frac{1}{N}\right) \cdots\left(1-\frac{s-1}{N}\right) .
$$

As long as $s \ll N$, this reduces to $V^{s}$, but it is important to note that if $N$ is small, then the large $N$ limit is not applicable and many results derived for gaseous behaviour, that implicitly take the large $N$ limit, are inapplicable to few body systems.

It is, of course, well-known that only the equation for $f_{1 \ldots N}$ is closed so that the exact time dependence of the system is determined completely by that equation and only by that equation. It has been, and still is, the object of considerable research to find approximate closed equations for the loworder density operators, which in turn determine all the possibly measured quantities of the system.

Eqs.(1)-(5) are first-order differential equations in the time, so that formal solutions can be immediately written down, appropriate for initial data at time $t_{0}$ :

$$
\begin{gathered}
f_{1}(t)=e^{-L_{1}\left(t-t_{0}\right)} f_{1}\left(t_{0}\right) \\
+n \operatorname{Tr}_{2} \int_{t_{0}}^{t} d t^{\prime} e^{-L_{1}\left(t-t^{\prime}\right)} \Theta_{12} f_{12}\left(t^{\prime}\right) \\
f_{12}(t)=e^{-L_{12}\left(t-t_{0}\right)} f_{12}\left(t_{0}\right) \\
+n \operatorname{Tr}_{3} \int_{t_{0}}^{t} d t^{\prime} e^{-L_{12}\left(t-t^{\prime}\right)}\left[\Theta_{13}+\Theta_{23}\right] f_{123}\left(t^{\prime}\right) \\
f_{123}(t)=e^{-L_{123}\left(t-t_{0}\right)} f_{123}\left(t_{0}\right) \\
+n \operatorname{Tr}_{4} \int_{t_{0}}^{t} d t^{\prime} e^{-L_{123}\left(t-t^{\prime}\right)}\left[\Theta_{14}+\Theta_{24}+\Theta_{34}\right] f_{1234}\left(t^{\prime}\right)
\end{gathered}
$$




$$
\begin{gathered}
f_{1 \ldots s}(t)=e^{-L_{1 \ldots s}\left(t-t_{0}\right)} f_{1 \ldots s}\left(t_{0}\right) \\
+n \operatorname{Tr}_{s+1} \int_{t_{0}}^{t} d t^{\prime} e^{-L_{1 \cdots s}\left(t-t^{\prime}\right)} \sum_{j=1}^{s} \Theta_{j, s+1} f_{1 \cdots s+1}\left(t^{\prime}\right) \\
\ldots \ldots \\
f_{1 \ldots N}(t)=e^{-L_{1 \cdots N}\left(t-t_{0}\right)} f_{1 \cdots N}\left(t_{0}\right) .
\end{gathered}
$$

The integral equations for the different reduced density operators can be combined. In particular, the integral equation for $f_{1}$ depends on $f_{12}$, which is itself given by an integral equation in terms of $f_{123}$. On inserting the $f_{12}$ integral equation into that for $f_{1}$ we find

$$
\begin{aligned}
f_{1}(t)= & e^{-L_{1}\left(t-t_{0}\right)} f_{1}\left(t_{0}\right) \\
& +n \operatorname{Tr}_{2} \int_{t_{0}}^{t} d t^{\prime} e^{-L_{1}\left(t-t^{\prime}\right)} \Theta_{12} e^{-L_{12}\left(t^{\prime}-t_{0}\right)} f_{12}\left(t_{0}\right) \\
& +n^{2} \operatorname{Tr}_{23} \int_{t_{0}}^{t} d t^{\prime} \int_{t_{0}}^{t^{\prime}} d t^{\prime \prime} e^{-L_{1}\left(t-t^{\prime}\right)} \Theta_{12} e^{-L_{12}\left(t^{\prime}-t^{\prime \prime}\right)} \\
& \times\left[\Theta_{13}+\Theta_{23}\right] f_{123}\left(t^{\prime \prime}\right)
\end{aligned}
$$

The differential form of Eq.(13), equivalently the direct substitution of Eq.(9) into Eq.(1), provides a means of classifying how the singlet density operator evolves, explicitly showing the role of free motion, pair interactions and three (or more) particle effects

$$
\begin{aligned}
\left(\frac{\partial}{\partial t}\right. & \left.+L_{1}\right) f_{1}(t) \\
& =n \operatorname{Tr}_{2} \Theta_{12} e^{-L_{12}\left(t-t_{0}\right)} f_{12}\left(t_{0}\right) \\
& +n^{2} \operatorname{Tr}_{23} \int_{t_{0}}^{t} d t^{\prime} \Theta_{12} e^{-L_{12}\left(t-t^{\prime}\right)}\left[\Theta_{13}+\Theta_{23}\right] f_{123}\left(t^{\prime}\right) .
\end{aligned}
$$

Eqs.(13) and (14) are exact results. The detailed nature of the term involving $f_{123}$ is not completely clear, since in general it includes the consequences of 
successive binary collision events and also the possibility of three (or more) particle collisions [by which is meant that all three particles are simultaneously interacting]. Indeed an aspect of this article is to clarify what parts of the exact evolution of the singlet are retained when solving the Boltzmann equation, and which are dropped. For this purpose it is useful to consider the structure of the series which arises from iterating the BBGKY hierarchy to arbitrary order. The present treatment is limited to the explicit form associated with keeping at most three-particle contributions.

Since the object of this article is to look at the relation between the BBGKY hierarchy and the Boltzmann equation, which inherently assumes that all gas properties are determined by the singlet density operator, it is appropriate to require that the initial state of the gas at time $t_{0}$ is completely described by the singlet $f\left(t_{0}\right)$, which means that the $N$-particle density operator is completely factorized, with the consequence that the lower ordered density operators $(s \ll N)$ satisfy

$$
f_{1 \ldots s}\left(t_{0}\right)=\prod_{i=1}^{s} f_{i}\left(t_{0}\right)
$$

Note how this is inconsistent with Eq.(7) if $s$ and $N$ are of similar magnitude, with the consequence that any equation deduced using Eq.(15) can only be valid for large $(N \gg 1)$ systems. In the Boltzmann equation binary collision effects are described by a pair transition superoperator $T$. This is the limit of the time dependent $T(t)$ superoperator (for particles 1 and 2) as

$$
T_{12}(t) \equiv \Theta_{12} e^{-L_{12} t} e^{K_{12} t} \underset{t \rightarrow \infty}{\longrightarrow} T_{12} .
$$

(Note that, by its definition, $T$ is not simply the commutator of the usual T-matrix with what follows.) As a precursor to identifying the contribution of a binary collision, the time dependent $T(t)$ can be seen to arise naturally, 
so this association is stressed in the following. With these remarks, Eq.(13), with Eq.(10) used to relate the three particle term to its value at $t_{0}$, can be rewritten as

$$
\begin{aligned}
f_{1}(t)= & e^{-L_{1}\left(t-t_{0}\right)} f_{1}\left(t_{0}\right) \\
& +n \operatorname{Tr}_{2} \int_{t_{0}}^{t} e^{-K_{12}\left(t-t^{\prime}\right)} T_{12}\left(t^{\prime}-t_{0}\right) e^{-K_{12}\left(t^{\prime}-t_{0}\right)} f_{1}\left(t_{0}\right) f_{2}\left(t_{0}\right) d t^{\prime} \\
& +n^{2} \operatorname{Tr}_{23} \int_{t_{0}}^{t} d t^{\prime} \int_{t_{0}}^{t^{\prime}} d t^{\prime \prime} e^{-K_{123}\left(t-t^{\prime}\right)} T_{12}\left(t^{\prime}-t^{\prime \prime}\right) e^{-K_{123}\left(t^{\prime}-t^{\prime \prime}\right)} \\
& \times\left[\Theta_{13}+\Theta_{23}\right] e^{-L_{123}\left(t^{\prime \prime}-t_{0}\right)} f_{1}\left(t_{0}\right) f_{2}\left(t_{0}\right) f_{3}\left(t_{0}\right)+\cdots
\end{aligned}
$$

It is emphasized that the use of the factorization at $t_{0}$ implies that this expression is exact as far as three particle effects are concerned in that all terms not explicitly displayed involve four or more particles. One interpretation of this equation, truncated at the three particle term, would be that the system has only three particles. This is technically not a reasonable interpretation since the numerical factors for the various terms involving powers of the gas density $n$ are only valid if $N$ is large, contrast the normalizations of Eq.(7) and Eq.(15). The implication, for the three particle term, is that particle 1 has interacted with two other particles in the $(N$-particle) system between the initial time " $t_{0}$ " and the present time " $t$ ", but the two other particles could be any pair of particles in the $N$ particle system.

To make contact between the three particle terms in Eq.(17) and binary collisions it is necessary to rewrite the evolution associated with $L_{123}$ in terms of the time dependent pair transition superoperator $T(t)$. For this purpose introduce $X(t) \equiv e^{\left(L_{13}+K_{2}\right) t} e^{-L_{123} t}$, which on differentiating and integrating, can be written as the integral equation

$$
e^{-L_{123} t}=e^{-\left(L_{13}+K_{2}\right) t} X(t)=e^{-\left(L_{13}+K_{2}\right)\left(t-t_{0}\right)} e^{-L_{123} t_{0}}
$$




$$
+\int_{t_{0}}^{t} d t^{\prime} e^{-\left(L_{13}+K_{2}\right)\left(t-t^{\prime}\right)}\left[\Theta_{12}+\Theta_{23}\right] e^{-L_{123} t^{\prime}} .
$$

Inserting the definition of $T(t)$ gives

$$
\begin{aligned}
\Theta_{13} e^{-L_{123}\left(t-t_{0}\right)} & =T_{13}\left(t-t_{0}\right) e^{-K_{123}\left(t-t_{0}\right)} \\
& +\int_{t_{0}}^{t} d t^{\prime} T_{13}\left(t-t^{\prime}\right) e^{-K_{123}\left(t-t^{\prime}\right)}\left[\Theta_{12}+\Theta_{23}\right] e^{-L_{123}\left(t^{\prime}-t_{0}\right)}
\end{aligned}
$$

whose first iteration yields

$$
\begin{aligned}
& \Theta_{13} e^{-L_{123}\left(t-t_{0}\right)}=T_{13}\left(t-t_{0}\right) e^{-K_{123}\left(t-t_{0}\right)} \\
& +\int_{t_{0}}^{t} d t^{\prime} T_{13}\left(t-t^{\prime}\right) e^{-K_{123}\left(t-t^{\prime}\right)}\left[T_{12}\left(t^{\prime}-t_{0}\right)+T_{23}\left(t^{\prime}-t_{0}\right)\right] e^{-K_{123}\left(t^{\prime}-t_{0}\right)} \\
& +\int_{t_{0}}^{t} d t^{\prime} \int_{t_{0}}^{t^{\prime}} d t^{\prime \prime} T_{13}\left(t-t^{\prime}\right) e^{-K_{123}\left(t-t^{\prime}\right)}\left[T_{12}\left(t^{\prime}-t^{\prime \prime}\right) e^{-K_{123}\left(t^{\prime}-t^{\prime \prime}\right)}\left(\Theta_{13}+\Theta_{23}\right)\right. \\
& \left.+T_{23}\left(t^{\prime}-t^{\prime \prime}\right) e^{-K_{123}\left(t^{\prime}-t^{\prime \prime}\right)}\left(\Theta_{12}+\Theta_{13}\right)\right] e^{-L_{123}\left(t^{\prime \prime}-t_{0}\right)}
\end{aligned}
$$

It follows that the three particle contribution to $f_{1}(t)$ may be rewritten as

$$
\begin{aligned}
\left.f_{1}(t)\right|_{3} \text { part. }= & n^{2} \operatorname{Tr}_{2,3} \int_{t_{0}}^{t} d t^{\prime} \int_{t_{0}}^{t^{\prime}} d t^{\prime \prime} e^{-K_{123}\left(t-t^{\prime}\right)} T_{12}\left(t^{\prime}-t^{\prime \prime}\right) e^{-K_{123}\left(t^{\prime}-t^{\prime \prime}\right)} \\
& \times\left[T_{13}\left(t^{\prime \prime}-t_{0}\right)+T_{23}\left(t^{\prime \prime}-t_{0}\right)\right] e^{-K_{123}\left(t^{\prime \prime}-t_{0}\right)} f_{1}\left(t_{0}\right) f_{2}\left(t_{0}\right) f_{3}\left(t_{0}\right) \\
+ & n^{2} \operatorname{Tr}_{23} \int_{t_{0}}^{t} d t^{\prime} \int_{t_{0}}^{t^{\prime}} d t^{\prime \prime} \int_{t_{0}}^{t^{\prime \prime}} d t^{\prime \prime \prime} e^{-K_{123}\left(t-t^{\prime}\right)} T_{12}\left(t^{\prime}-t^{\prime \prime}\right) e^{-K_{123}\left(t^{\prime}-t^{\prime \prime}\right)} \\
& \times\left\{T_{13}\left(t^{\prime \prime}-t^{\prime \prime \prime}\right) e^{-K_{123}\left(t^{\prime \prime}-t^{\prime \prime \prime}\right)}\left[T_{12}\left(t^{\prime \prime \prime}-t_{0}\right)+T_{23}\left(t^{\prime \prime \prime}-t_{0}\right)\right]\right. \\
& \left.+T_{23}\left(t^{\prime \prime}-t^{\prime \prime \prime}\right) e^{-K_{123}\left(t^{\prime \prime}-t^{\prime \prime \prime}\right)}\left[T_{12}\left(t^{\prime \prime \prime}-t_{0}\right)+T_{13}\left(t^{\prime \prime \prime}-t_{0}\right)\right]\right\} \\
& \times e^{-K_{123}\left(t^{\prime \prime \prime}-t_{0}\right)} f_{1}\left(t_{0}\right) f_{2}\left(t_{0}\right) f_{3}\left(t_{0}\right) \\
+n^{2} \operatorname{Tr}_{2,3} & \int_{t_{0}}^{t} d t^{\prime} \int_{t_{0}}^{t^{\prime}} d t^{\prime \prime} \int_{t_{0}}^{t^{\prime \prime}} d t^{\prime \prime \prime} \int_{t_{0}}^{t^{\prime \prime \prime}} d t_{4} e^{-K_{123}\left(t-t^{\prime}\right)} T_{12}\left(t^{\prime}-t^{\prime \prime}\right) e^{-K_{123}\left(t^{\prime}-t^{\prime \prime}\right)} \\
\times & \left\{T _ { 1 3 } ( t ^ { \prime \prime } - t ^ { \prime \prime \prime } ) e ^ { - K _ { 1 2 3 } ( t ^ { \prime \prime } - t ^ { \prime \prime \prime } ) } \left[T_{12}\left(t^{\prime \prime \prime}-t_{4}\right) e^{-K_{123}\left(t^{\prime \prime \prime}-t_{4}\right)}\left[\Theta_{13}+\Theta_{23}\right]\right.\right. \\
& \left.+T_{23}\left(t^{\prime \prime \prime}-t_{4}\right) e^{-K_{123}\left(t^{\prime \prime \prime}-t_{4}\right)}\left[\Theta_{12}+\Theta_{13}\right]\right] \\
+ & T_{23}\left(t^{\prime \prime}-t^{\prime \prime \prime}\right) e^{-K_{123}\left(t^{\prime \prime}-t^{\prime \prime \prime}\right)}\left[T_{12}\left(t^{\prime \prime \prime}-t_{4}\right) e^{-K_{123}\left(t^{\prime \prime \prime}-t_{4}\right)}\left[\Theta_{13}+\Theta_{23}\right]\right. \\
& \left.\left.+T_{13}\left(t^{\prime \prime \prime}-t_{4}\right) e^{-K_{123}\left(t^{\prime \prime \prime}-t_{4}\right)}\left[\Theta_{12}+\Theta_{23}\right]\right]\right\} \\
& \times e^{-L_{123}\left(t_{4}-t_{0}\right)} f_{1}\left(t_{0}\right) f_{2}\left(t_{0}\right) f_{3}\left(t_{0}\right) .
\end{aligned}
$$


If each $T(t)$ factor is interpreted as a binary collision, then the integral equation for $e^{-L_{123} t}$ has introduced more binary collisions between the three particles. Further iteration leads to an infinite series of binary collisions. But the structure of these terms shows that all but the first integral involves "recollisions" such as $T_{12} T_{13} T_{12}$ or "collision cycles" such as $T_{12} T_{13} T_{23}$. Actually, only for the contribution involving at most three particles at a time, the fourth binary collision must by necessity involve a recollision of at least one of the pair of particles. The terms in Eq.(17) involving four or more particles have an analogous structure.

Equation (21) and its further iteration, as well as the analogous expressions involving more particles can be interpreted as a binary collision expansion of the evolution superoperator. More correctly it is a binary transition expansion valid whenever the interaction potential is pairwise additive. The same type of expansion ${ }^{18}$, usually in energy representation (Fourier transform of the time series used here and for wavefunctions rather than the density operators), is the basis of multiparticle collision theory, see for example Newton ${ }^{19}$. In those applications one has in mind that all particles are within the range (restricting the discussion to non-relativistic mechanics) of the intermolecular potential (assumed pairwise additive) of all other particles. For the Boltzmann equation application that is to be discussed in this paper, binary collisions are thought of as independent events involving at most two particles within their range of interaction (this is what is referred to in the following as an isolated collision). The restriction of Eq.(21) and its analogs to include only isolated collisions is one of the main differences between the iterated solution of the BBGKY hierarchy and the iterated solution of the Boltzmann equation. Again, if the particles are considered to be classical rigid spheres, then the collision is instantaneous and the above distinction is 
unnecessary. But for realistic potentials it is always an important question as to whether the conditions are appropriate that collisions may be treated as isolated. These topics are discussed further in Section IV.

\section{The Quantum Boltzmann Equation}

The essence of the Boltzmann equation is that it describes the evolution of the one particle density operator for a dilute gas, which evolution is due to the free motion of the particles and isolated binary collisions between the particles. In this paper a quantum mechanical formalism is used but as far as the statistical arguments are concerned, the classical mechanical treatment is the same. Justification of the Boltzmann equation from first principles has been, and still is, a major problem with strongly held differences in opinion as to the validity of each treatment. The authors ${ }^{1-4}$ of the BBGKY hierarchy were the first to make this attempt. Except for the Green's function approach described by Kadanoff and Baym ${ }^{5}$ (which involves a closure ansatz for the self-energy, but will not be discussed further in this paper), all modern treatments are based on the BBGKY hierarchy. Bogoliubov's functional assumption $^{1}$ has played a major role in many attempts ${ }^{10-17}$ to justify the Boltzmann equation and to generalize it to higher density. While the details of the various treatments differ, all discussions leading to the Boltzmann equation must reduce to the inclusion of isolated binary collisions and free motion of the particles between successive isolated binary collisions. Section II has already discussed how the evolution of the singlet density operator as rigorously deduced from the BBGKY hierarchy can be expressed in terms of free motion and binary transition superoperators. But also stressed there, is that this structure does not in itself imply that the collisions are isolated. 
Further conditions are required. At low density it is expected that the collisions are isolated. Cohen ${ }^{10}$ expands in terms of independently evolving clusters of particles to obtain and generalize the Boltzmann equation. M.S. Green $^{11}$ approaches the derivation of the Boltzmann equation in the same manner but also discusses the problem of resummation of the series so that the isolated binary collisions involve the (classical) distribution functions at the time of collision, in contradistinction to treating collisions only during the initial time interval. Lanford ${ }^{12}$ has made a concerted effort to mathematically justify the Boltzmann equation. He bases his argument on Grad's ${ }^{20}$ limit, which interestingly takes the gas density to infinity rather than to zero as would be expected as a low density limit, while the size of the particles (rigid spheres) goes to zero in such a manner that the mean free path is finite while the second virial coefficient vanishes. In spite of much effort, Lanford is able to justify the Boltzmann equation only for times up to a fraction of the mean free time.

The present paper addresses the relation between two approaches to the "derivation" of the Boltzmann equation from the BBGKY hierarchy. Clearly these approaches are non-rigorous and depend on certain assumptions about the structure of the two and three particle density operators. Our interest is in the properties of real molecular systems in which the presence of soft intermolecular forces requires a clear understanding of how binary collisions become isolated. In this regard the present treatment has greater complexity than the oft discussed treatment of classical rigid spheres. A quantum formulation is used for the presentation but as it is assumed that no bound states are present, the formulation could be interpreted classically. The two "derivations" are reviewed in this section. In the following section (IV) the iterated solution of the Boltzmann equation is compared with the iterated 
solution of the BBGKY hierarchy with emphasis on their differences. Section IV also discusses how the assumed forms for the two and three particle density operators introduced in this section are consistent with the iterated solution of the Boltzmann equation.

The Approach Using Molecular Chaos: Boltzmann's original derivation of the equation that bears his name involved certain assumptions about the form of how (binary) collisions affect the evolution of the singlet (classical) distribution function"21. Key to his "Stosszahlansatz" is the assumption that the two particles about to collide are statistically independent. It is this molecular chaos assumption that has been used in the classic derivations ${ }^{1-3}$ of the Boltzmann equation from the BBGKY hierarchy and taken over in the quantum case $^{7}$ as well. The latter starts with the first BBGKY equation and, consistent with the notion of an isolated binary collision, assumes that the pair density operator that enters into this equation evolves during a collision according to the two particle quantum Liouville equation, Eq.(2) without the three particle term. On the basis that there is a time $t_{1}$ before the collision began and after each of the pair of particles has finished all previous collisions, the first BBGKY equation can be approximated by

$$
\begin{aligned}
& \left(\frac{\partial}{\partial t}+L_{1}\right) f_{1}(t) \\
& \quad=n \operatorname{Tr}_{2} \Theta_{12} e^{-L_{12}\left(t-t_{1}\right)} e^{K_{12}\left(t-t_{1}\right)} e^{-K_{12}\left(t-t_{1}\right)} f_{12}\left(t_{1}\right),
\end{aligned}
$$

where the factor $e^{-K_{12}\left(t-t_{1}\right)}$ and its inverse have been inserted before $f_{12}\left(t_{1}\right)$ in the last line. Note that $t_{1}$ has a significantly different meaning from the initial time $t_{0}$. Following Boltzmann it is assumed that the two-particle density operator factors at the time $t_{1}$ before the pair of particles has started 
to interact (collide). This is written

$$
f_{12}\left(t_{1}\right)=f_{1}\left(t_{1}\right) f_{2}\left(t_{1}\right)
$$

which, together with Eqs.(16) and (22) gives

$$
\left(\frac{\partial}{\partial t}+L_{1}\right) f_{1}(t)=n \operatorname{Tr}_{2} T_{12}\left(t-t_{1}\right) e^{-K_{12}\left(t-t_{1}\right)} f_{1}\left(t_{1}\right) f_{2}\left(t_{1}\right)
$$

Eq.(8) shows how the one-body distribution evolves in time. Eliminating the possibility that either particle of the pair $\{12\}$ interact with a third particle, so that the pair interaction is a truly binary collision event, we may write $f_{1}(t)=e^{-L_{1}\left(t-t_{1}\right)} f_{1}\left(t_{1}\right)$ for the purpose of substitution back into Eq.(24); this gives the quantum Boltzmann equation except for a time dependent transition superoperator

$$
\left(\frac{\partial}{\partial t}+L_{1}\right) f_{1}(t)=n \operatorname{Tr}_{2} T_{12}\left(t-t_{1}\right) f_{1}(t) f_{2}(t) .
$$

Finally, in the limit that $t-t_{1}$ is much larger than the duration of a collision, $T_{12}\left(t-t_{1}\right)$ can be replaced by its infinite time limit $T_{12}$, the transition superoperator, to give the quantum Boltzmann equation

$$
\left(\frac{\partial}{\partial t}+L_{1}\right) f_{1}(t)=n \operatorname{Tr}_{2} T_{12} f_{1}(t) f_{2}(t) .
$$

In review, this derivation has involved four separate steps: (a) dropping the three particle term in Eq.(14), applied for $t_{0}$ replaced by $t_{1}$; (b) the assumption of molecular chaos, Eq.(23), which allows one to write $f_{12}\left(t_{1}\right)=$ $f_{1}\left(t_{1}\right) f_{2}\left(t_{1}\right)$; (c) the replacement of $e^{-L_{1}\left(t-t_{1}\right)} f_{1}\left(t_{1}\right)$ by $f_{1}(t)$ in the collision term of Eq.(24), an approximation we will refer to as the "time-shift approximation"; and (d) the replacement of the time-dependent transition superoperator by its infinite time limit. Note that, although the time of the 
factorization of the pair density operator is just before the present collision, the solution $f_{1}$ of the Boltzmann equation (26) will describe a past history that includes successive pair collisions over times back to the initial time $t_{0}$ when the gas began to evolve. This feature is considered in more detail when the iterative solution of the Boltzmann equation is discussed in Sec. IV and compared with the iteration of the BBGKY hierarchy.

The Approach of Boercker and Dufty: In contrast to assuming a factorization of the pair density operator before a binary collision begins, Boecker and $\mathrm{Dufty}^{8}$ require such a factorization only at the initial time $t_{0}$. It is interpreted here that this time $t_{0}$ is the time at which the gas started to evolve so the factorization acts as a restriction on the class of initial states. But they also make a "binary collision approximation", which involves an assumed form for the three-particle density operator. Boercker and Dufty ${ }^{8}$ (BD) and later Dufty and $\mathrm{Kim}^{13}$ note that the second BBGKY equation involves terms such as $\Theta_{13} f_{123}(t)$. This vanishes unless particles 1 and 3 are interacting, in which case, for an isolated binary collision, particle 2 should be independent of (read "uncorrelated from") particles 1 and 3. This they implement by assuming that, in such a case, $f_{123}(t)$ factors into $f_{2}(t) f_{13}(t)$. Their treatment is actually more complicated because they require their density operators to have the proper Fermi-Dirac or Bose-Einstein statistics rather than the Boltzmann statistics assumed here for simplicity. Use of the factorization just mentioned in the two $f_{123}$ terms on the right side of Eq.(14) results in

$$
\begin{aligned}
& \left(\frac{\partial}{\partial t}+L_{1}\right) f_{1}(t)=n \operatorname{Tr}_{2} \Theta_{12} e^{-L_{12}\left(t-t_{0}\right)} f_{12}\left(t_{0}\right) \\
& \quad+n^{2} \operatorname{Tr}_{23} \int_{t_{0}}^{t} d t^{\prime} \Theta_{12} e^{-L_{12}\left(t-t^{\prime}\right)}\left(\Theta_{13} f_{13}\left(t^{\prime}\right) f_{2}\left(t^{\prime}\right)+\Theta_{23} f_{23}\left(t^{\prime}\right) f_{1}\left(t^{\prime}\right)\right)
\end{aligned}
$$

(Note that $t_{0}$ is the initial time.) BD then use the first BBGKY equation (1) 
to eliminate the two-body density operators, such as

$$
n \operatorname{Tr}_{3} \Theta_{13} f_{13}(t)=\left(\frac{\partial}{\partial t}+L_{1}\right) f_{1}(t) .
$$

The $\Theta_{23}$ term is treated similarly so that Eq.(27) becomes

$$
\begin{aligned}
& \left(\frac{\partial}{\partial t}+L_{1}\right) f_{1}(t)=n \operatorname{Tr}_{2} \Theta_{12} e^{-L_{12}\left(t-t_{0}\right)} f_{12}\left(t_{0}\right) \\
& \quad+n \operatorname{Tr}_{2} \int_{t_{0}}^{t} d t^{\prime} \Theta_{12} e^{-L_{12}\left(t-t^{\prime}\right)}\left(\frac{\partial}{\partial t^{\prime}}+K_{12}\right) f_{1}\left(t^{\prime}\right) f_{2}\left(t^{\prime}\right)
\end{aligned}
$$

The BD approach continues by carrying out the $t^{\prime}$ integral by parts. This is simplified by first introducing exponentials to remove the free motion superoperator $K_{12}$, equivalent to using the interaction representation. These calculations are as follows:

$$
\begin{aligned}
n \operatorname{Tr}_{2} & \int_{t_{0}}^{t} d t^{\prime} \Theta_{12} e^{-L_{12}\left(t-t^{\prime}\right)}\left(\frac{\partial}{\partial t^{\prime}}+K_{12}\right) f_{1}\left(t^{\prime}\right) f_{2}\left(t^{\prime}\right) \\
& =n \operatorname{Tr}_{2} \int_{t_{0}}^{t} d t^{\prime} \Theta_{12} e^{-L_{12}\left(t-t^{\prime}\right)} e^{K_{12}\left(t-t^{\prime}\right)} \frac{\partial}{\partial t^{\prime}}\left[e^{-K_{12}\left(t-t^{\prime}\right)} f_{1}\left(t^{\prime}\right) f_{2}\left(t^{\prime}\right)\right] \\
& =n \operatorname{Tr}_{2} \Theta_{12}\left[f_{1}(t) f_{2}(t)-e^{-L_{12}\left(t-t_{0}\right)} f_{1}\left(t_{0}\right) f_{2}\left(t_{0}\right)\right] \\
& -n \operatorname{Tr}_{2} \int_{t_{0}}^{t} d t^{\prime} \Theta_{12} \frac{\partial}{\partial t^{\prime}}\left[e^{-L_{12}\left(t-t^{\prime}\right)} e^{K_{12}\left(t-t^{\prime}\right)}\right] e^{-K_{12}\left(t-t^{\prime}\right)} f_{1}\left(t^{\prime}\right) f_{2}\left(t^{\prime}\right) .
\end{aligned}
$$

At this point $\mathrm{BD}$ also make the time-shift approximation [defined after Eq.(26)] in the last element of Eq.(30) by writing $e^{-K_{12}\left(t-t^{\prime}\right)} f_{1}\left(t^{\prime}\right) f_{2}\left(t^{\prime}\right)=$ $f_{1}(t) f_{2}(t)$. They argue that the error in this is small because the other factors in the last term in Eq.(30) limit the time integration to small $t-t^{\prime}$. On the other hand a glance at Eq.(8) indicates that what might be left out in the time shift approximation could be just one order higher in the density $n$. We will show, on the contrary, that a consistent application of the BD binary collision approximation, together with an assumption that pair collisions are instantaneous implies that this error vanishes. 
Once the time-shift approximation has been made the final integral in Eq.(30) is trivial and Eq.(29) becomes

$$
\left(\frac{\partial}{\partial t}+L_{1}\right) f_{1}(t)=n \operatorname{Tr}_{2} \Theta_{12} e^{-L_{12}\left(t-t_{0}\right)} e^{K_{12}\left(t-t_{0}\right)} f_{1}(t) f_{2}(t) .
$$

If $t-t_{0}$ is large enough we can again identify the combination of superoperators as the transition superoperator $T_{12}$, so that the WS equation (26) is obtained. In summary, Boercker and Dufty's approach involves three approximations: (a) the factorization of the product of the potential commutator and the three particle density operator according to $\Theta_{13} f_{123}=\Theta_{13} f_{13} f_{2}$; (b) the time-shift approximation; and (c) the replacement of the time-dependent transition superoperator by its infinite time limit. Note that while no molecular chaos is assumed in their approach, and $f_{12}$ is eliminated completely, the binary collision approximation has played a role analogous to that of molecular chaos. The relation between molecular chaos and the BD binary collision approximation is discussed further in the next section in terms of the iterated solutions of these equations and commented upon further in the summary section.

\section{Iterative Series Solutions of the Boltzmann Equation}

The exact iterative solution of the BBGKY hierarchy has been discussed in section II. This was expressed as a series involving increasing numbers of particles and increasing numbers of pair particle (time dependent) transition superoperators $T(t)$. The iterative solution of the Boltzmann equation is now examined and compared with the BBGKY result to understand the consequences of the assumptions that go into the derivation of the Boltzmann equation. These are first discussed for the approach using molecular chaos to derive the Boltzmann equation, and then for the Boercker Dufty approach. 
A formal integration of the Boltzmann equation (26) from an initial time $t_{0}$ gives

$$
f_{1}(t)=e^{-L_{1}\left(t-t_{0}\right)} f_{1}\left(t_{0}\right)+n \operatorname{Tr}_{2} \int_{t_{0}}^{t} d t^{\prime} e^{-K_{12}\left(t-t^{\prime}\right)} T_{12} f_{1}\left(t^{\prime}\right) f_{2}\left(t^{\prime}\right) .
$$

Iteration yields the infinite series

$$
\begin{aligned}
f_{1}(t)= & e^{-L_{1}\left(t-t_{0}\right)} f_{1}\left(t_{0}\right)+n \operatorname{Tr}_{2} \int_{t_{0}}^{t} d t^{\prime} e^{-K_{12}\left(t-t^{\prime}\right)} T_{12} e^{-K_{12}\left(t^{\prime}-t_{0}\right)} f_{1}\left(t_{0}\right) f_{2}\left(t_{0}\right) \\
& +n^{2} \operatorname{Tr}_{23} \int_{t_{0}}^{t} d t^{\prime} \int_{t_{0}}^{t^{\prime}} d t^{\prime \prime} e^{-K_{123}\left(t-t^{\prime}\right)} T_{12} e^{-K_{123}\left(t^{\prime}-t^{\prime \prime}\right)} \\
& \quad \times\left[T_{13}+T_{23}\right] e^{-K_{123}\left(t^{\prime \prime}-t_{0}\right)} f_{1}\left(t_{0}\right) f_{2}\left(t_{0}\right) f_{3}\left(t_{0}\right) \\
& +\cdots
\end{aligned}
$$

We have used the superoperator property $\operatorname{Tr}_{3}\left(e^{-L_{3} t} A\right)=\operatorname{Tr}_{3}(A)$ for any operator $A$ to insert $e^{-L_{3} t}$ in several places in this equation.

This series shows explicitly how the WS equation, as a generalized Boltzmann equation represents, for $t_{0}$ in the distant past, a large number of successive binary collisions. For example, the last term shown in Eq.(33) represents free propagation of three particles $\left(\right.$ via $\left.e^{-K_{123}\left(t^{\prime \prime}-t_{0}\right)}\right)$ from the initial time $t_{0}$ to $t^{\prime \prime}$ where a $\{23\}$-collision occurs; then there is free propagation to $t^{\prime}$ where a $\{12\}$-collision occurs; then free propagation again to time $t$. Terms of order $n^{k}$ represent $k$ successive binary collisions. It is important to emphasize that this equation implies that $f_{1}(t)$ is density dependent. This can be illustrated for a homogeneous system (so that all free motion, superoperator $K_{1 \ldots s}$ effects, can be dropped) by modelling the collision superoperator as a constant $T=-\sigma\langle v\rangle / V$ in terms of a effective cross section $\sigma$, mean relative velocity $\langle v\rangle$ and a factor $1 / V$ because collisions are localized. In this case the infinite series, Eq.(33), reduces to the infinite series expansion of an exponential 
decay toward equilibrium

$$
e^{-\left(t-t_{0}\right) / \tau}=\sum_{k} \frac{(-1)^{k}\left(t-t_{0}\right)^{k}}{k ! \tau^{k}}
$$

with relaxation rate $1 / \tau=-n V T=n \sigma\langle v\rangle$, using the normalization (7) of $f_{j}(t)$. Note that in this case the Boltzmann equation (26) technically reduces to

$$
\frac{\partial}{\partial t} f_{1}(t)=n T f_{1}(t) \operatorname{Tr}_{2} f_{2}(t)=n T f_{1}(t) V
$$

All discussions about the Boltzmann equation emphasize that it is valid only at low density. Most (all?) "derivations" of the Boltzmann equation from first principles take this over to interpret the Boltzmann equation as the first order result of a "density expansion" of the Liouville equation, using the BBGKY hierarchy as an intermediary in this expansion. But since $f_{1}$ is itself density dependent, any such emphasis on making a density expansion must be made more explicit. Clearly it is at least required that the singlet density operator $f_{1}$ be constant in this "expansion".

Comparison with the iterated solution of the BBGKY hierarchy, Eq.(17) with the more detailed form of the three particle contribution given by Eq.(21), shows that no recollisions or collision cycles occur in the iterated solution (33) of the Boltzmann equation. Essentially the Boltzmann equation assumes that no two particles ever collide a second time, and that correlations built up by collisions are never passed on to subsequently colliding particles by, for example, collision cycles. Effectively, the gaseous system is viewed as an infinite system so that once two particles have collided, the probability of them colliding again, or even influencing each other's subsequent evolution, is negligible. This is the essence of the molecular chaos assumption; the particles are always treated as being statistically uncorrelated before collision. 
The other obvious point of comparison is that the transition superoperator in the iteration of the Boltzmann equation is the time-independent, limiting form of the time-dependent transition superoperator that appears in the iteration of the BBGKY equation. Of the four steps discussed in Sec. III as being the basis of the derivation of the Boltzmann equation using the molecular chaos approach, points (b) and (d) have been mentioned. More subtle are the aspects associated with dropping the three particle term in the first BBGKY equation and the validity of the time-shift approximation. A proposed interpretation of what these two approximations have accomplished is to consider that they are part of implementing the complete separation of the evolution into free motion and instantaneous binary collisions. Dropping the three particle term in Eq.(14), for $t_{0}$ replaced by $t_{1}$, is incumbent on the short time period $t-t_{1}$ for which this equation is to be applied. Since the pair $\{12\}$ have merely had time to enter into a binary collision, there is inherently no time for a third particle to interact with either of the pair and retain the restriction that only binary collisions occur. The possibility that a prior binary collision could be described by the presence of $\Theta_{13}$ or $\Theta_{23}$ contradicts the definition of $t_{1}$ as the time just before the binary collision. Thus the three particle term is dropped in the molecular chaos approach. The timeshift approximation is related to the notion that the particles evolve freely between collisions. The presence of time dependent transition superoperators also allows for the possibility of a very short time between when a particle interacts with first one and then a second particle, this again allowing for the possibility of a true three particle collision rather than restricting the evolution associated with three particles to involving only successive isolated binary collisions. Eliminating this possibility emphasizes the need to treat collisions as instantaneous if only isolated binary collisions are to contribute 
to the evolution of the gas. The classical description of this separation of time scales is to take the mean free time $\tau=1 /(n \sigma\langle v\rangle)$ to be much larger than the time $\tau_{\text {coll }}=d /\langle v\rangle$ of duration of a collision $(d$ is the effective molecular diameter). For classical rigid spheres where $\sigma \approx d^{2}$ this ratio is equivalent to $\tau_{\text {coll }} / \tau \approx n d^{3} \ll 1$. This also immediately implies that the second virial coefficient for the gas is to vanish so the gas is ideal.

A more careful analysis of the dimensionless quantities upon which the iterated solution (33) of the Boltzmann equation depends and how it differs from the iterated solution, Eq.(17) together with Eq.(21), of the BBGKY hierarchy is important for understanding the differences between the two iterations. Neglecting the detailed effects of the free motion, the iterated solution of the Boltzmann equation depends solely on the finiteness of $t / \tau=$ $n \sigma\langle v\rangle t=-n V T t$. Analysis of the three particle contribution (21) to the iterated solution of the BBGKY hierarchy shows that the first term involves $n^{2} T^{2} V^{2} t^{2} \approx(t / \tau)^{2}$ while the second term involves $n^{2} T^{3} V^{2} t^{3} \approx(t / \tau)^{2}(T t)$, and the third term contains even higher powers of $T t$. This simple analysis has set all transition superoperators as a common constant and ignored any constraints on the time integrals except for their explicitly displayed limits. Within this analysis it is seen that the absence in the Boltzmann solution of all but the first term is equivalent, for finite (large) times, to the fact that the range of the potential is negligible compared to the size of the system. If the time actually does get large enough so that Poincaré recurrence effects are important, then $T t$ can be finite, such terms must be retained in the BBGKY solution, and of course the Boltzmann equation is inapplicable. Even for classical rigid spheres the analysis is not so simple. For a recollision to occur, the two particles involved in the third collision must be directed at each other. This can occur only for a small set of phase points if the time 
is long $^{22}$ and is responsible for the divergence !!!!CHECK!!! of certain time correlation functions. An analysis ${ }^{22}$ of the phase space region that contributes to recollisions and collision cycles implies that these are small if $n d^{3}$ is small. This implies that the BBGKY solution can be well approximated by the Boltzmann equation solution only if $n d^{3}$ is small. While classical rigid spheres can only recollide and be involved in collision cycles, real particles with their soft potentials can undergo real three particle collisions, that is, all three particles are simultaneously within each others range of interaction. Even the first term in Eq.(21) has contributions from such effects while the Boltzmann solution (33) does not. If one assumes the time $\left(t^{\prime}-t^{\prime \prime}\right)$ between the action of the two $T$ superoperators is of the order of the time $d /\langle v\rangle$ taken for a particle to traverse its potential range, then the contribution to this true three particle collision is $n^{2} T^{2} V^{2} t d /\langle v\rangle \approx(t / \tau) d /(\langle v\rangle \tau)$. The latter factor, $d /(\langle v\rangle \tau) \approx n d \sigma$ is the analog of the rigid sphere $n d^{3}$, so again, if $n d^{3}$ is small, true three particle collisions can be ignored and the BBGKY solution contains only the effect of isolated binary collisions. There is still the difference between the series in that the Boltzmann solution involves a time independent transition superoperator while in the BBGKY solution, the transition superoperator is time dependent. But if $n d \sigma=t_{\text {coll }} / \tau$ is small, then typically the time $\left(t^{\prime}-t^{\prime \prime}\right)$, see the first term in Eq.(21), between collisions is to be long compared to the time of duration of a collision so that the compensating free and full motions in $T_{12}\left(t^{\prime}-t^{\prime \prime}\right)$ have sufficient time so that the transition superoperator reaches it asymptotic, time independent limit $T$ of Eq.(16). It may be remarked that the limit transition superoperator is spatially delocalized, that is, the two particles are not required to be at the same spatial position, an important aspect when there are internal spin states present (ignored in this paper), so that there can be a transfer of angular momentum from internal state 
spin to the translational degrees of freedom, which is responsible for spin relaxation ${ }^{23}$. This delocalized property of the collision is also responsible for density corrections to the transport coefficients ${ }^{24}$. Such corrections are proportional to $n d^{3}$ so they should not be treated in isolation, but rather the kinetic theory should be extended beyond the Boltzmann equation to include collision cycles and recollisions as well as true three particle collisions and corrections associated with the fact that the binary transition superoperator cannot be treated in its asymptotic limit. Except for the binary collision delocalization correction, only the crudest estimates ${ }^{28}$ of these effects have been made for particles other than classical rigid spheres.

Lanford $^{12}$ compares the properties of the Boltzmann equation with the BBGKY hierarchy by examining the structure of a comparable (Boltzmann) hierarchy. In terms of the present (quantum) formalism Lanford's approach is to define a set of $s$-particle density operators $F_{1 \ldots s}(t) \equiv \prod_{j} f_{j}(t)$, where each singlet density operator $f_{j}(t)$ for particle $j$ satisfies the Boltzmann equation. From the Boltzmann equation (26), it immediately follows that

$$
\left(\frac{\partial}{\partial t}+K_{1 \ldots s}\right) F_{1 \ldots s}(t)=n \operatorname{Tr}_{s+1} \sum_{j=1}^{s} T_{j, s+1} F_{1 \ldots s+1}(t) .
$$

This typical equation of the Boltzmann hierarchy differs from the corresponding equation (4) in the BBGKY hierarchy by the replacement of $L_{1 \ldots s}$ by $K_{1 \ldots s}$ and of $\Theta_{j, s+1}$ by $T_{j, s+1}$. The latter clearly indicates that in the Boltzmann hierarchy, coupling between different members of the hierarchy occur by isolated binary collisions, whereas in the BBGKY hierarchy, coupling is by the interparticle potential. More subtle is the fact that each particle of the $s$-particles described by $F_{1 \ldots s}$ evolves freely in the Boltzmann hierarchy, while in the BBGKY hierarchy, all s-particles interact among each other. The absence of all the interaction terms between the $s$-particles is equivalent 
to the lack of recollisions and collision cycles in the Boltzmann equation. These contrasting properties of Eqs. (4) and (36) emphasize the basic difference between the predictions of the Boltzmann equation and the Liouville equation.

The Boercker Dufty binary collision approximation involves approximating the structure of $\Theta_{13} f_{123}\left(t^{\prime}\right)$ and analogously for particles $\{23\}$; see Eq.(27). It is also relevant to note that this is to be used only when $\Theta_{12} e^{-L_{12}\left(t-t^{\prime}\right)}$ acts on the result; compare Eqs.(13), (14) or (27) where the specific labels for the times vary from one equation to another. The exact evolution of $f_{123}(t)$ is given by the integrated form of the third BBGKY equation (10) and, restricting the discussion to the purely three particle contribution, entirely via $e^{-L_{123}\left(t-t_{0}\right)}$. This in turn has been expanded in terms of time dependent pair transition superoperators and with the interaction $\Theta_{13}$ is given by Eq.(19). Explicitly this gives after iteration as in Eq.(20)

$$
\begin{gathered}
\left.\Theta_{13} f_{123}(t)\right|_{3} \text { part. } \\
=T_{13}\left(t-t_{0}\right) e^{-K_{123}\left(t-t_{0}\right)} f_{123}\left(t_{0}\right) \\
+\int_{t_{0}}^{t} d t^{\prime} T_{13}\left(t-t^{\prime}\right) e^{-K_{123}\left(t-t^{\prime}\right)}\left[T_{12}\left(t^{\prime}-t_{0}\right)+T_{23}\left(t^{\prime}-t_{0}\right)\right] e^{-K_{123}\left(t^{\prime}-t_{0}\right)} f_{123}\left(t_{0}\right) \\
+\int_{t_{0}}^{t} d t^{\prime} \int_{t_{0}}^{t^{\prime}} d t^{\prime \prime} T_{13}\left(t-t^{\prime}\right) e^{-K_{123}\left(t-t^{\prime}\right)}\left[T_{12}\left(t^{\prime}-t^{\prime \prime}\right) e^{-K_{123}\left(t^{\prime}-t^{\prime \prime}\right)}\left(\Theta_{13}+\Theta_{23}\right)\right. \\
\left.+T_{23}\left(t^{\prime}-t^{\prime \prime}\right) e^{-K_{123}\left(t^{\prime}-t^{\prime \prime}\right)}\left(\Theta_{12}+\Theta_{13}\right)\right] e^{-L_{123}\left(t^{\prime \prime}-t_{0}\right)} f_{123}\left(t_{0}\right)
\end{gathered}
$$

Operating on this with the interaction between particles $\{12\}$ and taking the limit in each transition superoperator gives

$$
\begin{aligned}
& \left.\Theta_{12} e^{-L_{12}\left(t-t^{\prime}\right)} \Theta_{13} f_{123}\left(t^{\prime}\right)\right|_{3} \text { part. } \rightarrow T_{12} e^{-K_{12}\left(t-t^{\prime}\right)} T_{13} e^{-K_{123}\left(t^{\prime}-t_{0}\right)} f_{123}\left(t_{0}\right) \\
& +\int_{t_{0}}^{t^{\prime}} d t^{\prime \prime} T_{12} e^{-K_{12}\left(t-t^{\prime}\right)} T_{13} e^{-K_{123}\left(t^{\prime}-t^{\prime \prime}\right)}\left[T_{12}+T_{23}\right] e^{-K_{123}\left(t^{\prime \prime}-t_{0}\right)} f_{123}\left(t_{0}\right) \\
& +\int_{t_{0}}^{t^{\prime}} d t^{\prime \prime} \int_{t_{0}}^{t^{\prime \prime}} d t^{\prime \prime \prime} T_{12} e^{-K_{12}\left(t-t^{\prime}\right)} T_{13} e^{-K_{123}\left(t^{\prime}-t^{\prime \prime}\right)}\left[T_{12} e^{-K_{123}\left(t^{\prime \prime}-t^{\prime \prime \prime}\right)}\left(\Theta_{13}+\Theta_{23}\right)\right. \\
& \left.+T_{23} e^{-K_{123}\left(t^{\prime \prime}-t^{\prime \prime \prime}\right)}\left(\Theta_{12}+\Theta_{13}\right)\right] e^{-L_{123}\left(t^{\prime \prime \prime}-t_{0}\right)} f_{123}\left(t_{0}\right)
\end{aligned}
$$


It is now a matter of dropping all recollision and collision cycle terms, together with the introduction of the assumed initial factorization, to obtain

$$
\begin{aligned}
& \left.\Theta_{12} e^{-L_{12}\left(t-t^{\prime}\right)} \Theta_{13} f_{123}\left(t^{\prime}\right)\right|_{3} \text { part. } \\
& \quad \rightarrow T_{12} e^{-K_{12}\left(t-t^{\prime}\right)} T_{13} e^{-K_{123}\left(t^{\prime}-t_{0}\right)} f_{1}\left(t_{0}\right) f_{2}\left(t_{0}\right) f_{3}\left(t_{0}\right) .
\end{aligned}
$$

This can be recognized as the three particle contribution from the product $\Theta_{12} e^{-L_{12}\left(t-t^{\prime}\right)} \Theta_{13} f_{13}\left(t^{\prime}\right) f_{2}\left(t^{\prime}\right)$, namely from the Boercker Dufty binary collision approximation, subject to the same conditions of transition superoperator limit and factorization at $t_{0}$. Thus it is seen that the Boercker Dufty approach is an alternative way of selecting out only isolated binary collisions for the evolution of the singlet density operator. An attempt to given a more physical rationalization of the BD approach is made in Sec. VII.

\section{The Correction to the Boltzmann Equation}

The validity of the Boltzmann equation is discussed in this section by examining the difference between the exact evolution of the singlet density operator and its evolution as given by the Boltzmann equation.

Using Eq.(15) one can write $f_{12}\left(t_{0}\right)=f_{1}\left(t_{0}\right) f_{2}\left(t_{0}\right)$ in the first term of Eq. (14). Next solve Eq.(8) for the first term on the right:

$$
e^{-L_{1}\left(t-t_{0}\right)} f_{1}\left(t_{0}\right)=f_{1}(t)-n \operatorname{Tr}_{3} \int_{t_{0}}^{t} d t^{\prime} e^{-L_{1}\left(t-t^{\prime}\right)} \Theta_{13} f_{13}\left(t^{\prime}\right)
$$


Substitute this result for the factors $f_{i}\left(t_{0}\right)$ that now appear in Eq.(14) to give

$$
\begin{aligned}
\left(\frac{\partial}{\partial t}+L_{1}\right) f_{1}( & t)=n \operatorname{Tr}_{2} \Theta_{12} e^{-L_{12}\left(t-t_{0}\right)} e^{K_{12}\left(t-t_{0}\right)} \\
\times & {\left[f_{1}(t)-n \operatorname{Tr}_{3} \int_{t_{0}}^{t} d t^{\prime} e^{-L_{1}\left(t-t^{\prime}\right)} \Theta_{13} f_{13}\left(t^{\prime}\right)\right] } \\
\times & {\left[f_{2}(t)-n \operatorname{Tr}_{4} \int_{t_{0}}^{t} d t^{\prime} e^{-L_{2}\left(t-t^{\prime}\right)} \Theta_{24} f_{24}\left(t^{\prime}\right)\right] } \\
+ & n^{2} \operatorname{Tr}_{23} \Theta_{12} \int_{t_{0}}^{t} d t^{\prime} e^{-L_{12}\left(t-t^{\prime}\right)}\left[\Theta_{13}+\Theta_{23}\right] f_{123}\left(t^{\prime}\right) \\
=n \operatorname{Tr}_{2} & T_{12}\left(t-t_{0}\right) f_{1}(t) f_{2}(t)+G(t),
\end{aligned}
$$

where $G(t)$ is defined by

$$
\begin{aligned}
G(t)= & -n^{2} \operatorname{Tr}_{23} T_{12}\left(t-t_{0}\right) f_{1}(t) \int_{t_{0}}^{t} d t^{\prime} e^{-L_{2}\left(t-t^{\prime}\right)} \Theta_{23} f_{23}\left(t^{\prime}\right) \\
& -n^{2} \operatorname{Tr}_{23} T_{12}\left(t-t_{0}\right) \int_{t_{0}}^{t} d t^{\prime} e^{-L_{1}\left(t-t^{\prime}\right)} \Theta_{13} f_{13}\left(t^{\prime}\right) f_{2}(t) \\
& +n^{3} \operatorname{Tr}_{234} T_{12}\left(t-t_{0}\right) \int_{t_{0}}^{t} d t^{\prime} e^{-L_{1}\left(t-t^{\prime}\right)} \Theta_{13} f_{13}\left(t^{\prime}\right) \\
& \times \int_{t_{0}}^{t} d t^{\prime \prime} e^{-L_{2}\left(t-t^{\prime \prime}\right)} \Theta_{24} f_{24}\left(t^{\prime \prime}\right) \\
& +n^{2} \operatorname{Tr}_{23} \Theta_{12} \int_{t_{0}}^{t} d t^{\prime} e^{-L_{12}\left(t-t^{\prime}\right)}\left[\Theta_{13}+\Theta_{23}\right] f_{123}\left(t^{\prime}\right) .
\end{aligned}
$$

The Boltzmann equation differs from Eq.(41) in two ways, first is the absence of $G(t)$ which will be called for the present purposes the correction to the Boltzmann equation, and second in the requirement that the infinite time limit in the transition superoperator must be taken. If the limit is not taken, then as previously discussed, this could allow particles $\{12\}$ to be interacting while particle 1 and/or 2 are still interacting with some other particle in the gas, essentially allowing three (or more) particle collisions. Thus one can see the need for this limit in any rationalization of the Boltzmann equation; this will be henceforth assumed. What this process does is to remove the possibility of simultaneous three body collisions by making all binary collision events appear as instantaneous events. However, there remain other events 
involving correlations among three particles; the recollision and collision cycle events discussed in the iteration procedure of Sec.II are of this nature. $G(t)$ will describe such events and there remains the discussion of how these are removed in the molecular chaos and BD approaches.

In the molecular chaos approach to deriving the Boltzmann equation $G(t)$ is seen to necessarily involve three particles. Thus this term contributes three particle effects to the rate of change of the singlet, so should be dropped in a theory that is to be based on allowing only isolated binary collisions. We return to this point after discussing the Boercker-Dufty approach to explicitly show the connection between $G(t)$ and the three-particle collision term that arises in generalizing the Boltzmann equation ${ }^{10-16}$ to higher density. For the Boercker-Dufty approach, it is now shown that $G(t)$ vanishes when their "binary collision approximation" and the infinite time limit of transition superoperators is taken, thus showing that the Boltzmann equation is derived in a consistent manner in the BD approach.

The BD approximation ${ }^{13,8}$ would seem to be ideally designed to minimize $G(t)$ since, for example, the first term of Eq.(42) seems to be approximating the three-body correlations by a factorization of the form $f_{1}(t) f_{23}\left(t^{\prime}\right)$. (Note, however, the times of the two elements are different.) Let us make the BD binary collision approximation in the $f_{123}$ term and see how complete is the cancellation of $G(t)$. That is, write in Eq.(42)

$$
\left[\Theta_{13}+\Theta_{23}\right] f_{123}\left(t^{\prime}\right)=\Theta_{13} f_{13}\left(t^{\prime}\right) f_{2}\left(t^{\prime}\right)+\Theta_{23} f_{23}\left(t^{\prime}\right) f_{1}\left(t^{\prime}\right)
$$

Make use of Eq.(8) to write $f_{1}(t)$ in terms of $f_{1}\left(t^{\prime}\right)$ at time $t^{\prime}$ to give for the 
first term in Eq.(42)

$$
\begin{aligned}
- & n^{2} \operatorname{Tr}_{23} T_{12}\left(t-t_{0}\right) \int_{t_{0}}^{t} d t^{\prime}\left[e^{-L_{1}\left(t-t^{\prime}\right)} f_{1}\left(t^{\prime}\right)\right. \\
& \left.+n \operatorname{Tr}_{4} \int_{t^{\prime}}^{t} d t^{\prime \prime} e^{-L_{1}\left(t-t^{\prime \prime}\right)} \Theta_{14} f_{14}\left(t^{\prime \prime}\right)\right] e^{-L_{2}\left(t-t^{\prime}\right)} \Theta_{23} f_{23}\left(t^{\prime}\right) \\
= & -n^{2} \operatorname{Tr}_{23} T_{12}\left(t-t_{0}\right) \int_{t_{0}}^{t} d t^{\prime} e^{-K_{12}\left(t-t^{\prime}\right)} \Theta_{23} f_{23}\left(t^{\prime}\right) f_{1}\left(t^{\prime}\right) \\
& -n^{3} \operatorname{Tr}_{234} T_{12}\left(t-t_{0}\right) \int_{t_{0}}^{t} d t^{\prime} \int_{t^{\prime}}^{t} d t^{\prime \prime} e^{-L_{1}\left(t-t^{\prime \prime}\right)} \Theta_{14} f_{14}\left(t^{\prime \prime}\right) e^{-L_{2}\left(t-t^{\prime}\right)} \Theta_{23} f_{23}\left(t^{\prime}\right) .
\end{aligned}
$$

(Note the lower limit in the inner integral in the last line.) The second term in $G(t)$ is handled in the same way:

$$
\begin{aligned}
& -n^{2} \operatorname{Tr}_{23} T_{12}\left(t-t_{0}\right) \int_{t_{0}}^{t} d t^{\prime} e^{-L_{1}\left(t-t^{\prime}\right)} \Theta_{13} f_{13}\left(t^{\prime}\right) \\
& \quad \times\left[e^{-L_{2}\left(t-t^{\prime}\right)} f_{2}\left(t^{\prime}\right)+n \operatorname{Tr}_{4} \int_{t^{\prime}}^{t} d t^{\prime \prime} e^{-L_{2}\left(t-t^{\prime \prime}\right)} \Theta_{24} f_{24}\left(t^{\prime \prime}\right)\right] \\
& =-n^{2} \operatorname{Tr}_{23} T_{12}\left(t-t_{0}\right) \int_{t_{0}}^{t} d t^{\prime} e^{-K_{12}\left(t-t^{\prime}\right)} \Theta_{13} f_{13}\left(t^{\prime}\right) f_{2}\left(t^{\prime}\right) \\
& \quad-n^{3} \operatorname{Tr}_{234} T_{12}\left(t-t_{0}\right) \int_{t_{0}}^{t} d t^{\prime} \int_{t^{\prime}}^{t} d t^{\prime \prime} e^{-L_{1}\left(t-t^{\prime}\right)} \Theta_{13} f_{13}\left(t^{\prime}\right) e^{-L_{2}\left(t-t^{\prime \prime}\right)} \Theta_{24} f_{24}\left(t^{\prime \prime}\right) .
\end{aligned}
$$

Thus the BD approximation implies that the correction term $G(t)$ becomes

$$
\begin{aligned}
& G^{(B D K)}(t)=-n^{2} \operatorname{Tr}_{23} T_{12}\left(t-t_{0}\right) \int_{t_{0}}^{t} d t^{\prime} e^{-K_{12}\left(t-t^{\prime}\right)} \Theta_{23} f_{23}\left(t^{\prime}\right) f_{1}\left(t^{\prime}\right) \\
& -n^{2} \operatorname{Tr}_{23} T_{12}\left(t-t_{0}\right) \int_{t_{0}}^{t} d t^{\prime} e^{-K_{12}\left(t-t^{\prime}\right)} \Theta_{13} f_{13}\left(t^{\prime}\right) f_{2}\left(t^{\prime}\right) \\
& +n^{2} \operatorname{Tr}_{23} \int_{t_{0}}^{t} d t^{\prime} T_{12}\left(t-t^{\prime}\right) e^{-K_{12}\left(t-t^{\prime}\right)}\left[\Theta_{13} f_{13}\left(t^{\prime}\right) f_{2}\left(t^{\prime}\right)+\Theta_{23} f_{23}\left(t^{\prime}\right) f_{1}\left(t^{\prime}\right)\right] \\
& +n^{3} \operatorname{Tr}_{234} T_{12}\left(t-t_{0}\right) \int_{t_{0}}^{t} d t^{\prime} \int_{t_{0}}^{t} d t^{\prime \prime} e^{-L_{1}\left(t-t^{\prime}\right)} \Theta_{13} f_{13}\left(t^{\prime}\right) e^{-L_{2}\left(t-t^{\prime \prime}\right)} \Theta_{24} f_{24}\left(t^{\prime \prime}\right) \\
& -n^{3} \operatorname{Tr}_{234} T_{12}\left(t-t_{0}\right) \int_{t_{0}}^{t} d t^{\prime} \int_{t^{\prime}}^{t} d t^{\prime \prime}\left[e^{-L_{1}\left(t-t^{\prime \prime}\right)} \Theta_{14} f_{14}\left(t^{\prime \prime}\right) e^{-L_{2}\left(t-t^{\prime}\right)} \Theta_{23} f_{23}\left(t^{\prime}\right)\right. \\
& \left.+e^{-L_{1}\left(t-t^{\prime}\right)} \Theta_{13} f_{13}\left(t^{\prime}\right) e^{-L_{2}\left(t-t^{\prime \prime}\right)} \Theta_{24} f_{24}\left(t^{\prime \prime}\right)\right] .
\end{aligned}
$$

If we take the infinite time limit of $T(t)$ wherever it appears, the first two traces cancel against the third. Combine the two parts of the last trace by 
interchanging indices 3 and 4 and times $t^{\prime}$ and $t^{\prime \prime}$ in the first part to give for the last trace

$$
\begin{gathered}
-n^{3} \operatorname{Tr}_{234} T_{12}\left(t-t_{0}\right)\left[\int_{t_{0}}^{t} d t^{\prime \prime} \int_{t^{\prime \prime}}^{t} d t^{\prime}+\int_{t_{0}}^{t} d t^{\prime} \int_{t^{\prime}}^{t} d t^{\prime \prime}\right] e^{-L_{1}\left(t-t^{\prime}\right)} \Theta_{13} f_{13}\left(t^{\prime}\right) \\
\times e^{-L_{2}\left(t-t^{\prime \prime}\right)} \Theta_{24} f_{24}\left(t^{\prime \prime}\right) .
\end{gathered}
$$

We see that the two integrals combine nicely to give $\int_{t_{0}}^{t} d t^{\prime} \int_{t_{0}}^{t} d t^{\prime \prime}$ which makes the sum precisely cancel against the fourth trace term, and $G^{(B D K)}(t)=0$. This result means that under the assumption that the $T$ operator is time independent - equivalent to the assumption that the collision duration time is much shorter than the mean-free path travel time, a reasonable assumption if the density is low - the use of the BD binary collision approximation in the derivation of the WS equation is internally consistent. No additional approximations are needed, in particular, the time-shift approximation that had to be made in Eq.(30) to get the WS equation does not appear as a separate approximation.

Finally, it is useful to explore the nature of the correction term $G(t)$ in more detail and explicitly show that it introduces three (and more) particle effects. For three particles we make the substitutions of the expressions for $f_{i j}$, and $f_{123}$ from Eqs.(9) and (10) - along with the initial factorization condition (15) in Eq.(42) for $G(t)$. Only those terms that involve three particle contributions are considered, being denoted by $G^{(3)}$ :

$$
\begin{aligned}
& G^{(3)}=-n^{2} \operatorname{Tr}_{23} T_{12}\left(t-t_{0}\right) f_{1}(t) \int_{t_{0}}^{t} d t^{\prime} e^{-L_{2}\left(t-t^{\prime}\right)} \Theta_{23} e^{-L_{23}\left(t^{\prime}-t_{0}\right)} f_{2}\left(t_{0}\right) f_{3}\left(t_{0}\right) \\
& -n^{2} \operatorname{Tr}_{23} T_{12}\left(t-t_{0}\right) \int_{t_{0}}^{t} d t^{\prime} e^{-L_{1}\left(t-t^{\prime}\right)} \Theta_{13} e^{-L_{13}\left(t^{\prime}-t_{0}\right)} f_{1}\left(t_{0}\right) f_{3}\left(t_{0}\right) f_{2}(t) \\
& \quad+n^{2} \operatorname{Tr}_{23} \Theta_{12} \int_{t_{0}}^{t} d t^{\prime} e^{-L_{12}\left(t-t^{\prime}\right)}\left[\Theta_{13}+\Theta_{23}\right] e^{-L_{123}\left(t^{\prime}-t_{0}\right)} f_{1}\left(t_{0}\right) f_{2}\left(t_{0}\right) f_{3}\left(t_{0}\right)
\end{aligned}
$$


To express this as a collision integral, the singlet density operators should be evaluated at time $t$. This can be accomplished by using the time-shift approximation. Of course there will be a correction to this but it will involve an extra particle so can be dropped when calculating $G^{(3)}$. We write, for example,

$$
f_{i}\left(t_{0}\right)=e^{L_{1}\left(t-t_{0}\right)} f_{i}(t)+O(n)
$$

Eq.(48) then becomes

$$
\begin{aligned}
& G^{(3)}=-n^{2} \operatorname{Tr}_{23} T_{12}\left(t-t_{0}\right) \int_{t_{0}}^{t} d t^{\prime}\left\{e^{-K_{23}\left(t-t^{\prime}\right)} \Theta_{23} e^{-L_{23}\left(t^{\prime}-t_{0}\right)} e^{K_{23}\left(t-t_{0}\right)}\right. \\
& \left.+e^{-K_{13}\left(t-t^{\prime}\right)} \Theta_{13} e^{-L_{13}\left(t^{\prime}-t_{0}\right)} e^{K_{13}\left(t-t_{0}\right)}\right\} f_{1}(t) f_{2}(t) f_{3}(t) \\
& +n^{2} \operatorname{Tr}_{23} \Theta_{12} \int_{t_{0}}^{t} d t^{\prime} e^{-L_{12}\left(t-t^{\prime}\right)}\left[\Theta_{13}+\Theta_{23}\right] \\
& \quad \times e^{-L_{123}\left(t^{\prime}-t_{0}\right)} e^{K_{123}\left(t^{\prime}-t_{0}\right)} f_{1}(t) f_{2}(t) f_{3}(t) \\
& =-n^{2} \operatorname{Tr}_{23} T_{12}\left(t-t_{0}\right)\left\{\int_{t_{0}}^{t} d t^{\prime} e^{-K_{23}\left(t-t_{0}\right)} \frac{\partial}{\partial t^{\prime}}\left(e^{K_{23}\left(t^{\prime}-t_{0}\right)} e^{-L_{23}\left(t^{\prime}-t_{0}\right)}\right) e^{K_{23}\left(t-t_{0}\right)}\right. \\
& \left.\quad+\int_{t_{0}}^{t} d t^{\prime} e^{-K_{13}\left(t-t_{0}\right)} \frac{\partial}{\partial t^{\prime}}\left(e^{K_{13}\left(t^{\prime}-t_{0}\right)} e^{-L_{13}\left(t^{\prime}-t_{0}\right)}\right) e^{K_{13}\left(t-t_{0}\right)}\right\} f_{1}(t) f_{2}(t) f_{3}(t) \\
& +n^{2} \operatorname{Tr}_{23} \Theta_{12} \int_{t_{0}}^{t} d t^{\prime} e^{-L_{12}\left(t-t_{0}\right)} \frac{\partial}{\partial t^{\prime}}\left(e^{L_{12}\left(t^{\prime}-t_{0}\right)} e^{-L_{123}\left(t^{\prime}-t_{0}\right)}\right) \\
& \times e^{K_{123}\left(t-t_{0}\right)} \frac{f_{1}(t) f_{2}(t) f_{3}(t)}{f_{1}}
\end{aligned}
$$

The integrals in this are easily done to give

$$
\begin{gathered}
G^{(3)}=n^{2} \operatorname{Tr}_{23} \Theta_{12}\left\{e^{-L_{123}\left(t-t_{0}\right)} e^{K_{123}\left(t-t_{0}\right)}-e^{-L_{12}\left(t-t_{0}\right)} e^{K_{123}\left(t-t_{0}\right)}\right. \\
-e^{-L_{12}\left(t-t_{0}\right)} e^{K_{12}\left(t-t_{0}\right)}\left[e^{-L_{23}\left(t-t_{0}\right)} e^{K_{23}\left(t-t_{0}\right)}-1\right. \\
\left.\left.\quad+e^{-L_{13}\left(t-t_{0}\right)} e^{K_{13}\left(t-t_{0}\right)}-1\right]\right\} f_{1}(t) f_{2}(t) f_{3}(t)
\end{gathered}
$$

In the limit of large $t-t_{0}$ the combinations of exponential superoperators become Møller superoperators, typically $\Omega_{12}=\lim _{t \rightarrow \infty} e^{-L_{12} t} e^{K_{12} t}$ for the pair $\{12\}$ and for the three-body Møller superoperator $\Omega_{123}=\lim _{t \rightarrow \infty} e^{-L_{123} t} e^{K_{123} t}$. This leads to

$$
G^{(3)}=n^{2} \operatorname{Tr}_{23} \Theta_{12}\left[\Omega_{123}-\Omega_{12}-\Omega_{12}\left(\Omega_{23}+\Omega_{13}-2\right)\right] f_{1}(t) f_{2}(t) f_{3}(t)
$$


which is just the standard result for the three-body collision integral ${ }^{10-16}$. Thus the Boltzmann equation involves the neglect of what is normally called three-body collisions. Without examining any details one can note that the recollision and collision cycles that made up the difference between the iteration series from the Boltzmann equation and that from the complete BBGKY hierarchy, for example in Eq.(21), must be included in the above three-body collision integral ${ }^{10-16}$.

\section{Klimontovich Binary Collision Approximation}

Klimontovich ${ }^{17}$ introduces the pair correlation function $g_{12}(t) \equiv$ $f_{12}(t)-f_{1}(t) f_{2}(t)$ and the three-particle correlation function $g_{123}(t) \equiv$ $f_{123}(t)-f_{1}(t) f_{2}(t) f_{3}(t)-f_{1}(t) g_{23}(t)-f_{1}(t) g_{13}(t)-f_{3}(t) g_{12}(t)$. The first two BBGKY equations are then rewritten as

$$
\left(\frac{\partial}{\partial t}+L_{1}^{e f f}(t)\right) f_{1}(t)=n \operatorname{Tr}_{2} \Theta_{12} g_{12}(t) \equiv I_{1}(t)
$$

and

$$
\begin{gathered}
\left(\frac{\partial}{\partial t}+L_{12}^{e f f}(t)\right) f_{12}(t)=n \operatorname{Tr}_{3}\left[\Theta_{13}+\Theta_{23}\right]\left[g_{123}(t)\right. \\
\left.+f_{1}(t) g_{23}(t)+f_{2}(t) g_{13}(t)\right]
\end{gathered}
$$

where the effective Liouville superoperators are

$$
L_{1}^{e f f}(t) \equiv L_{1}-n \operatorname{Tr}_{2} \Theta_{12} f_{2}(t)
$$

and

$$
L_{12}^{e f f}(t) \equiv L_{12}-n \operatorname{Tr}_{3}\left[\Theta_{13}+\Theta_{23}\right] f_{3}(t)
$$

These differ from the bare Liouville superoperators by the average force terms that can be of immense importance for plasma physics. As stated in the 
introduction, Klimontovich's work has been written here using a quantum language for convenience of comparison with Boercker and Dufty's work. It is easy to translate the formalism into classical terms by interpreting the particle labels as position and momentum labels, replacing any single particle trace by $h^{-3}$ times the integrals over the particle's position and momentum, and finally replacing any $f_{1 \ldots s}$ by $h^{3 s}$ times the corresponding phase space distribution function.

Klimontovich's method of deriving a Boltzmann equation is to introduce a binary collision approximation. This consists of dropping the $g_{123}$ term in Eq. (54) and keeping only those $g_{j 3}(t)$ terms in which the $\Theta_{i j}$ and $g_{i j}$ particle labels coincide. This reduces the trace in Eq.(54) to

$$
\begin{aligned}
& \operatorname{Tr}_{3}\left[\Theta_{13} g_{13}(t) f_{2}(t)+\Theta_{23} g_{23}(t) f_{1}(t)\right] \\
&=I_{1}(t) f_{2}(t)+I_{2}(t) f_{1}(t) \\
&=\left[\frac{\partial}{\partial t}+L_{1}^{e f f}(t)+L_{2}^{e f f}(t)\right] f_{1}(t) f_{2}(t) .
\end{aligned}
$$

After translating into classical mechanical terms and accounting for differences in notation this is Klimontovich's Eq.(2.18). This has the same form as Boercker and Dufty's binary collision approximation [see Eq.(29)] differing only in the replacement of $L_{i}$ by $L_{i}^{e f f}$ on both sides of the equation. (It may be useful to note that Klimontovich's equations are not exactly equivalent to those of Boercker and Dufty since the average force terms on each side of the equation act on different quantities and thus do not cancel.) The rationale for the BD approximation in terms of taking into successful binary collisions thus is seen to apply equally well to the Klimontovich approach, but of course the ordering of terms according to powers of $n d^{3}$ is different. The mean force terms are formally of higher order in $n d^{3}$, but for long range forces such as the Coulomb force, the effective range $d$ of the potential is infinite so this 
ordering is inappropriate. Rather, the mean force term accounts for the long range part of the potential and the collisions, expressed entirely in terms of particle correlations, are determined by the corresponding short range part of the potential.

\section{Summary}

Two methods of obtaining the quantum Boltzmann equation from the BBGKY hierarchy have been discussed. The first is based on the molecular chaos assumption in the form that two particles about to enter into a collision, are statistically independent. The second is a binary collision approximation introduced by Boercker and Dufty in which the three particle density operator factors into a product of a pair density operator and a singlet, depending on which pair of particles is interacting. While these approaches appear very different, both result in the Boltzmann equation. What is not expressly emphasized is that, in the process of deriving the Boltzmann equation in either approach, the infinite time limit of the transition superoperator is taken, Eq.(16), and, except when in the process of colliding, the particles evolve freely. The present work emphasizes that these added assumptions are crucial to the derivation of the Boltzmann equation for realistic potentials and are of at least equal importance to the molecular chaos, or alternately, the Boercker Dufty binary collision approximation. In most discussions of the derivation of the Boltzmann equation, these assumptions are ignored. Such assumptions are of course unnecessary for the treatment of classical rigid spheres, but realistic potentials include dispersion forces that vary as $1 / r^{6}$. These are short range compared to the Coulomb and dipolar forces that cause the equilibrium second virial coefficient to diverge, but formally 
the interaction never ends. Thus the separation into free and collisional motion is not an on-and-off affair but when a collision ends is a matter of degree. Whether this separation into free and collisional motion can be done is a major problem for realistic potentials. The Boltzmann equation assumes such a separation can be accomplished and formally appears as the ability to take the limit of the transition superoperator.

The limits of validity of the Boltzmann equation have been investigated by examining the series obtained by iterating the integral equation for the singlet density operator, arrived at by a formal time integration of the Boltzmann equation, and comparing this series with the corresponding series obtained from the exact BBGKY hierarchy. This was aided by expressing the formal solution of the BBGKY hierarchy in terms of time dependent pair transition superoperators and free evolution superoperators, by exact formal algebraic calculation. It is this representation of the exact behaviour of the singlet which looks most like the series derived from the Boltzmann equation.

The first obvious difference between the BBGKY derived series and that from the Boltzmann equation is that the latter involves only the time independent transition superoperator, correctly the infinite time limit of the time dependent transition superoperator. Taking this limit wherever it occurs in the BBGKY derived series implies that only isolated binary collisions occur. For example, it is easy to write out the time evolution superoperator $e^{-L_{123} t}$ for three interacting particles as an infinite series of time convolutions of two particle evolution superoperators; just iterate Eq.(18). Thus a threeparticle process in which all three particles are simultaneously interacting can be considered as a sequence of two body events but with arbitrary times between the pair interactions. Taking the infinite time limit for each pair breaks this possible interpretation and requires that each pair collision be an 
isolated event. We see this as "a", if not "the", crucial step in understanding the structure of the Boltzmann equation. It is also where irreversibility enters, or at least an arrow of time, because it is the transition superoperator for scattering in a positive time sense that is selected. The resulting series derived from the BBGKY hierarchy then involves isolated binary collisions (which formally appear as instantaneous events) and free motion between collisions.

The series derived from the Boltzmann equation is further limited by the fact that no recollisions or collision cycles appear. By having no recollisions is meant that, if particles labelled $\{i j\}$ collide at some time, then they never again collide. Collision cycles are exemplified by the sequence of collisions $\{i j\} \rightarrow\{i k\} \rightarrow\{j k\}$, allowing collisions between particles $\{i j\}$ and $\{i k\}$ to possibly influence the outcome of the $\{j k\}$ collsion. No such terms enter into the Boltzmann series of events, so that any derivation of the Boltzmann equation from the BBGKY hierarchy must eliminate such events in some manner. If these recollision and collision cycle events are eliminated from the BBGKY derived series, then it agrees completely with the Boltzmann derived series of events; only the requirements of isolated collision (infinite limit of the time dependent binary transition superoperators) and the removal of recollisions and collision cycles is needed to get the Boltzmann series from the BBGKY series. No other assumption such as molecular chaos is required. Of course the initial factorization of the density operator, Eq.(15), is interpreted as an initial condition on the quantum Liouville equation rather than an assumption about the dynamics of the $N$ particle system. The interpretation of the lack of recollisions and collision cycles is presumably that, in a dilute gas, the probability of such events are negligible so that they should be ignored. 
From the point of view of the two derivations of the Boltzmann equation discussed in this paper, molecular chaos clearly emphasizes that binary collision processes are to be isolated. But it should be a warning that if the infinite time limit is not taken, then there still remains the possibility that three particle collisions may be partly incorporated into the treatment. The "binary collision approximation" of Boercker and Dufty, and of Klimontovich, emphasizes which pair of particles of a triple of particles are colliding. Exactly how this is to be interpreted has been a difficulty for the present authors, and led to various discussions with Dufty. On the basis of the series derived from the BBGKY hierarchy and from iterating the BD binary collision approximation, we interpret that the collision to which their binary collision approximation is to be applied is the collision just before the present one. This is seen by the fact that in application, the BD binary collision approximation is to approximate the evolution for the pair particle density operator that is to be used in the first BBGKY equation. Specifically, if $f_{12}$ is to be used in the first BBGKY equation, then it is to be used for a collision between particles $\{12\}$. The second BBGKY equation, describing the evolution of $f_{12}$, involves $\Theta_{13} f_{123}$, whose approximation by $\Theta_{13} f_{13} f_{2}$ is then involved in approximating this term as a binary collision, the $\{13\}$ collision which preceeds the $\{12\}$ collision. Thus the BD binary collision approximation is describing the consequences of a past binary collision in order to predict the present course of events. Such an interpretation is novel, and for that matter there appears to be very little written on trying to interpret the BD binary collision approximation. We see this as effectively removing recollision and collision cycle events. As part of the BD approach, any explicit appearance of the pair density operator is subsequently removed by use of the first BBGKY equation to obtain an equation solely in terms of the singlet density opera- 
tor. We see again the need to take the infinite time limit of the transition superoperator in order to retain only isolated binary collision events. Thus we do not see, as Dufty and $\mathrm{Kim}^{13}$ claim, that their work "extends the familiar Waldmann-Snider equation to arbitrary length and time scales", rather we see their derivation of the WS (quantum Boltzmann) equation as an alternative to the usual approach using molecular chaos and giving the same result.

In this work the expansions have been classified according to the numbers of particles that contribute to a particular quantity. This is often thought of, and described as, a density expansion. We comment that the latter can be subject to misinterpretation. In particular, even the Boltzmann equation is nonlinear in the density $n$, so that its solution $f_{1}$ is a complicated function of the density. This is exemplified in an elementary manner by Eq.(34), which shows explicitly that the solution includes contributions of all orders in the density, essentially associated with the consequences of successive binary collisions. Thus any approximation of the BBGKY hierarchy by making a density expansion, to arrive at, for example the Boltzmann equation, must be careful to emphasize the conditions under which the expansion is to be carried out, for example, by keeping the singlet density operator $f_{1}$ constant.

As a final comment, we remark that the series expansions show that if corrections to the Boltzmann equation are to be considered, it is necessary to be cognizant that three particle collisions, binary recollision and collision cycle events all occur. One must also consider the time dependence of the binary transition superoperators. Attempts to consider the effect of one type of correction at a time are laudable but one must not lose sight of the composite structure of all possible corrections. Bogoliubov's method ${ }^{1}$ is often considered as giving a general procedure for generalizing the Boltzmann 
equation to arbitrary density. We look on Cohen's work ${ }^{10}$ as carrying out this program. But we also see the need for resumming the series to express the collisions in terms of distribution functions that arise from successive past collisions as Green ${ }^{11}$ has discussed. Generalizations of the two procedures are clearly possible. Hollinger and Curtiss ${ }^{25}$ introduced a molecular chaos assumption at the $s$ particle level and Lowry and Snider ${ }^{26}$ have approached the problem of chemical recombination and decay from this point of view. Unpublished work of one of the present authors has looked at generalizing the BD approach to factorizing the four particle density operator into the various possible types of collisions that occur involving at most three particles at a time. The present paper has not attempted to examine such questions, but clearly there are many possible approaches to generalizing the Boltzmann equation.

\section{Acknowledgments}

We would like to thank Professor James Dufty for useful correspondence. This work was supported in part by the Natural Sciences and Engineering Research Council of Canada and by NATO travel grant CRG 930644. Laboratoire Kastler Brossel est associé au CNRS UA18, à l'Universite Paris 6, et à l'ENS. 


\section{References}

1. N. Bogoliubov, J. Phys. (U.S.S.R.) 10:265 (1946); Problems of a Dynamical Theory in Statistical Physics, translated by E.K. Gora, in Studies in Statistical Physics, Vol. I, Part A, edited by J. de Boer and G.E. Uhlenbeck (North-Holland, Amsterdam, 1962).

2. M. Born and H.S. Green, Proc. Roy. Soc. London A188:10 (1946).

3. J.G. Kirkwood, J. Chem. Phys. 14:180 (1946).

4. J. Yvon, La Théorie Statistique des Fluides et l'Equation d'Etat, Act. scient. et ind. No. 203 (Hermann, Paris, 1935).

5. L.P. Kadanoff and G. Baym, Quantum Statistical Mechanics (W.A. Benjamin, Inc., New York, 1962).

6. L. Boltzmann, Wein. Ber. 66:275 (1872) (Collected Works, Vol. 1, p.315).

7. R.F. Snider, J. Chem. Phys. 32:1051 (1960); see R.F. Snider and B.C. Sanctuary, J. Chem. Phys. 55:1555 (1971) for the general properties associated with the presence of off-diagonal elements of the density matrix.

8. D.B. Boercker and J.W. Dufty, Ann. Phys. 119:43 (1979).

9. L. Waldmann, Z. Naturforsch. 12a:660 (1957).

10. E. D. G. Cohen, Fundamental Problems in Statistical Mechanics, Vol. II (North-Holland, Amsterdam, 1968). 
11. M.S. Green, J. Chem. Phys. 25, 836 (1956).

12. O.E. Lanford, in Dynamical Systems, Theory and Applications. J. Moser, ed. (Springer, New York, 1975), p. 1.

13. J.W. Dufty and C.S. Kim, preprint (1993).

14. J.H. Ferziger and H.G. Kaper, Mathematical Theory of Transport Processes in Gases (North-Holland, Amsterdam, 1972).

15. J. A. McLennan, Introduction to Nonequilibrium Statistical Mechanics (Prentice Hall, Englewood Cliffs, NJ, 1989).

16. E. M. Lifshitz and L.P. Pitaevskii, Physical Kinetics (Pergamon Press, Oxford, 1981), §17. (1990).

17. Yu.L. Klimontovich, Kinetic Theory of Nonideal Gases and Nonideal Plasmas (Pergamon Press, Oxford, 1982).

18. K.M. Watson, Phys. Rev. 89:575 (1953).

19. R.G. Newton, Scattering Theory of Waves and Particles (SpringerVerlag, New York, 1982).

20. H. Grad, in Handbuch der Physik, Vol. XII, S. Flügge, ed. (Springer, Berlin, 1958), p. 205.

21. For example, see D. ter Haar, Elements of Statistical Mechanics (Rinehart and C., Inc., New York, 1954) Appendix I.

22. M.S. Green and R.A. Piccirelli, Phys. Rev. 132:1388 (1963). !!!!! NOT RIGHT REFERENCE !!!! 
23. M.W. Thomas and R.F. Snider, J. Stat. Phys. 2:61 (1970).

24. R.F. Snider and C.F. Curtiss, Phys. Fluids 1:122 (1958); 3:903 (1960).

25. H.B. Hollinger and C.F. Curtiss, J. Chem. Phys. 33:1386 (1960).

26. J.T. Lowry and R.F. Snider, J. Chem. Phys. 61:2320 (1974).

27. F. Laloë and W. J. Mullin, J. Stat. Phys. 59, 725 (1990).

28. see J.C. Rainwater and D.G. Friend, Phys. Rev. A 36:4062 (1987) and references therein for a review of theories modelling the transport virials of real molecular gases. 